\title{
Características morfológicas em plantas de cultivares de aveia como indicadoras do potencial competitivo com plantas daninhas
}

\author{
Morphological traits in oat plants cultivars as indicators of competitive potential against weeds
}

\author{
Carlos Eduardo Schaedler ${ }^{\mathrm{I}}$ Nilson Gilberto Fleck ${ }^{\mathrm{II}}$ Fausto Borges Ferreira ${ }^{\mathrm{III}}$ \\ Carlos Alberto Lazaroto ${ }^{\mathrm{III}}$ Mauro Antônio Rizzardi ${ }^{\mathrm{IV}}$
}

\section{RESUMO}

\begin{abstract}
O objetivo do experimento foi comparar cultivares de aveia quanto a características morfológicas de plantas no início do desenvolvimento que possam mostrar relação com a capacidade de competir com plantas daninhas. Para tanto, foi realizado um experimento a campo na Universidade Federal do Rio Grande do Sul, em Eldorado do Sul, Rio Grande do Sul (RS), durante a estação fria de 2005. O delineamento experimental utilizado foi o de blocos completamente casualizados, com quatro repetições. Foram investigadas 10 cultivares de aveia, a saber: 'ALBASUL', 'CFT 1', 'FAPA 5', 'FAPA 6', 'UFRGS 19', 'UPF 16', 'UPFA 20', 'UPFA 22', 'URS 21'e 'URS 22'. As características de planta avaliadas foram: estatura de planta, matéria seca da parte aérea, cobertura foliar do solo, incrementos em estatura, matéria seca e cobertura, potencial competitivo, produtividade biológica, produtividade de grãos e índice de colheita. Constatou-se que cultivares de aveia mostram variações acentuadas em características morfológicas no início do ciclo. As cultivares 'UPF 16' e 'UPFA 22' apresentam características típicas de plantas competidoras devido à elevada cobertura proporcionada por seus dosséis e aos rápidos incrementos em estatura e cobertura do solo na fase inicial de desenvolvimento. A cultivar 'ALBASUL', por outro lado, não é portadora de atributos morfológicos que a caracterizem como portadora de perfil competidor com plantas concorrentes no início do ciclo, embora apresente elevada produtividade de grãos e elevado índice de colheita.
\end{abstract}

Palavras-chave: Avena sativa, interferência, competição, genótipos, manejo cultural.

\section{ABSTRACT}

The aim of this study was to compare plant oat morphological traits of cultivars at early stages, that may be more competitive against weeds. A field experiment was performed at Universidade Federal do Rio Grande do Sul, in Eldorado do Sul - RS, during 2005 cold season, in a completely random design, with four replicates. The oat cultivars evaluated were: 'ALBASUL', 'CFT 1', 'FAPA 5', 'FAPA 6', 'UFRGS 19', 'UPF 16', 'UPFA 20', 'UPFA 22', 'URS 21', and 'URS 22'. Plant traits evaluated were: plant height, shoot dry matter, soil canopy coverage, increases in height, dry matter and coverage, competitive potential, biological yield, grain yield, and harvest index. Oat cultivars show outstanding variations regarding to morphological traits during the initial growth period. 'UPF 16' and 'UPFA 22' cultivars present typical characteristics of competing plants, due to high soil coverage by their canopies and to fast increments in height and in soil coverage, during the initial growth period. 'ALBASUL' cultivar, on the other hand, does not hold a competitor profile with weeds at the beginning of development, although it presents high grain yield and high harvest index.

Key words: Avena sativa, interference, competition, genotypes, cultural management.

\section{INTRODUÇÃO}

A utilização de cultivares com elevada capacidade competitiva constitui-se em ferramenta importante para o manejo cultural de plantas daninhas. Entende-se por habilidade competitiva a aptidão ou capacidade da planta/cultura em levar vantagem na competição com seus vizinhos, suprimindo o crescimento de outra, ou sua habilidade de manter relativamente inalterado seu próprio crescimento na

'Departamento de Fitossanidade, Universidade Federal de Pelotas (UFPel), 96010-900, Pelotas, RS, Brasil. E-mail: caduschaedler@yahoo.com.br. Autor para correspondência.

"UFPel, Pelotas, RS, Brasil.

II'Universidade Federal do Rio Grande do Sul (UFRGS), Porto Alegre, RS, Brasil.

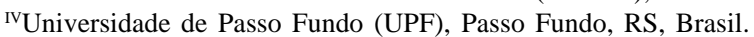


presença de competidores, evitando, desse modo, ser suprimida(GOLDBERG \& LANDA, 1991). Grande parte dos estudos de interferência de plantas daninhas em culturas considera somente aspectos da ocorrência e do impacto da competição na produtividade das culturas. Apenas um número restrito de estudos foi realizado para quantificar a importância de características de genótipos de culturas na definição de sua habilidade competitiva (RADOSEVICH et al., 1997; NI et al., 2000).

O potencial de competitividade de culturas com espécies daninhas geralmente aumenta por meio da cobertura precoce do solo proporcionada pelo dossel e da estatura elevada, principalmente na fase inicial de desenvolvimento (FLECK, 1980; BALBINOT JR. et al., 2003; HAEFELE et al., 2004). A interceptação de luz pelo dossel da comunidade vegetal depende, além da população e do arranjo das plantas, de um conjunto de características morfológicas, tais como: capacidade de afilhamento, estatura de planta, número de folhas formadas, área foliar, distribuição das folhas, ângulo foliar, grau de decumbência do limbo das folhas e produção de matéria seca da parte aérea (BLACKSHAW, 1994). Por exemplo, cultivares de trigo que possuíam maior estatura conseguiram sombrear as plantas daninhas, levando vantagem na competição (WICKS et al., 2004).

Nesse contexto, é importante investigar se ocorre variabilidade em características morfológicas de cultivares de aveia durante os estádios iniciais. Numa primeira etapa, o estudo é conduzido na ausência de plantas concorrentes, de modo a evitar que estas exerçam influências diferenciais sobre os genótipos em comparação. Em etapa posterior, as cultivares são submetidas à competição visando comprovar se o potencial dessas características é real e se, sob competição, a vantagem inicial positiva se sustenta durante todo o ciclo da cultura.

Devido ao rápido surgimento de casos de resistência de plantas daninhas aos herbicidas, a habilidade competitiva pode se tornar um critério ainda mais importante para a seleção de novas cultivares em relação àquelas priorizadas anteriormente em programas de melhoramento de plantas (LEMERLE et al., 2006). No contexto atual da agricultura, conhecer melhor as características de desenvolvimento inicial de cultivares de aveia que possam se associar à habilidade competitiva, a exemplo do que já ocorre com outras culturas anuais, é importante para acrescentar novas estratégias ao manejo integrado de plantas daninhas. Com isso, pode-se reduzir a quantidade de herbicidas utilizados e, consequentemente, minimizar os custos de produção e o impacto ambiental que muitas vezes eles provocam.
Desse modo, o objetivo do experimento foi avaliar a variabilidade de algumas características morfológicas em plantas de cultivares de aveia que sugerem associação com habilidade competitiva sobre plantas concorrentes no início do desenvolvimento das plantas.

\section{MATERIAL E MÉTODOS}

Foi conduzido um experimento em campo durante a estação fria de 2005, na Estação Experimental Agronômica da Universidade Federal do Rio Grande do Sul (EEA - UFRGS), localizada no Município de Eldorado do Sul, Rio Grande do Sul (RS). O delineamento experimental utilizado foi o de blocos casualizados, com quatro repetições. Cada unidade experimental constou de cinco fileiras de aveia com 3m de comprimento, espaçadas de 0,2m, sendo a área útil de $3 \mathrm{~m}^{2}$. No experimento foram utilizadas 10 cultivares de aveia, a saber: 'ALBASUL', 'CFT 1', 'FAPA 5', 'FAPA6', 'UFRGS 19', 'UPF 16', 'UPFA20', 'UPFA22’, 'URS 21', 'URS 22'.

A semeadura ocorreu no dia 23 de junho de 2005, em densidades entre 290 e 330 sementes $\mathrm{m}^{-2}$. A emergência das plantas foi acompanhada por meio de contagens realizadas aos oito e 16 dias após a semeadura, em 1,0m em duas fileiras por unidade experimental. As demais variáveis avaliadas no estudo foram: cobertura foliar do solo (CS), estatura de planta (EST), matéria seca da parte aérea (MSPA), potencial competitivo (PC), produtividade biológica aparente (PBA), produtividade de grãos (PG) e índice de colheita (ICA).

A CS pelas cultivares foi avaliada aos $16 \mathrm{e}$ 29 dias após a emergência (DAE), por meio dos métodos visual, fotográfico e de câmera de dossel. A CS e o PC foram realizados por dois avaliadores que atuaram independentemente. Para realizar as avaliações fotográficas, foram usadas duas áreas de $0,25 \mathrm{~m}^{2} \mathrm{em}$ cada unidade experimental. Com auxílio de programa computacional Sigma Scan, as fotos foram digitalizadas de modo a permitir a diferenciação da coloração da folhagem das plantas em relação à coloração do fundo. Posteriormente, foi estimada a participação da área ocupada pela parte aérea das plantas em relação à área total amostrada. Para avaliar CS pela câmera de dossel (First Growth Canopy Camera - Decagon), foram fotografadas duas áreas de $0,215 \mathrm{~m}^{2}$ em cada unidade experimental. A câmera fornecia automaticamente os valores de CS, expressos em porcentagem. Com base nos resultados fornecidos pelo método visual, foram estimados os incrementos em CS entre 16 e 29DAE. 
Para as determinações de EST e MSPA, foram escolhidas, ao acaso, 10 plantas por parcela. A EST foi obtida pela medida das plantas desde o nível do solo até o ápice destas, com o limbo foliar distendido. Para obtenção da MSPA, as plantas foram acondicionadas em sacos de papel e secas em estufa a $60^{\circ} \mathrm{C}$ até se obter massa constante, quando foram pesadas. Os resultados foram expressos em mg planta ${ }^{-1}$. O PC das cultivares, estimado quando surgiu o primeiro nó, baseou-se em escala proposta por LEMERLE et al. (2001). A avaliação foi realizada de forma visual, utilizando-se características de planta, tais como: estatura, vigor, largura e inclinação das folhas, afilhamento e cobertura. Para tal, seguiu-se o padrão de avaliação proposto, atribuindo-se as notas 1 , 3 e 5 às cultivares que apresentavam características como baixo, médio e alto $\mathrm{PC}$, respectivamente.

Para obtenção da PBA, foram selecionadas duas fileiras de aveia em área destinada à produtividade de grãos, onde foram coletadas cinco plantas, sequencialmente, em cada fileira, sendo medida a área de solo ocupada pela amostra. As plantas foram secas em estufa a $60^{\circ} \mathrm{C}$ até se obter massa constante, quando foram pesadas. A PBA compreendeu o somatório das massas das partes aéreas das plantas, incluindo grãos (cariopses). A divisão da massa dos grãos pela PBA forneceu o IC, expresso em porcentagem. Por ocasião da colheita das panículas, realizada 119 DAE, determinou-se a $\mathrm{PG}$ em área de $3 \mathrm{~m}^{2}$. Após pesagem dos grãos, foi determinada a umidade destes, e as massas foram corrigidas para o teor de $13 \%$.
Os dados obtidos para as variáveis avaliadas foram submetidos à análise de variância, por meio do teste F. Em caso de diferença significativa, as médias dos tratamentos foram comparadas aplicandose o teste de Duncan, adotando-se o nível de 5\% de probabilidade.

\section{RESULTADOS E DISCUSSÃO}

As cultivares 'UPF 16' e 'UPFA 22' apresentaram maiores coberturas pelos dosséis (Tabela 1). Por outro lado, as cultivares 'ALBASUL'e 'UFRGS 19’ geralmente figuraram como aquelas que propiciaram menores coberturas. Plantas que fornecem elevada cobertura, principalmente em estádios iniciais de desenvolvimento, em geral, demonstram maior competitividade com as infestantes (FLECK, 1980; BALBINOT JR. et al., 2003; HAEFELE et al., 2004). Para haver habilidade competitiva superior, por meio do sombreamento de culturas sobre plantas daninhas, também é necessário elevada EST, o que possibilita captação de energia luminosa no estrato superior do dossel (WICKS et al., 2004). Em relação aos graus de associação entre os métodos usados para a variável CS, verificaram-se valores mais elevados entre os métodos visual e fotográfico, tanto aos $16(\mathrm{r}=0,76)$, quanto aos 29DAE ( $\mathrm{r}=0,83)$. O método câmera de dossel associou-se melhor com o fotográfico aos 16DAE $(\mathrm{r}=0,48)$; aos 29DAE houve maior associação daquele método com o visual $(\mathrm{r}=0,67)$.

Tabela 1 - Cobertura do solo por plantas de aveia, avaliada por meio de três métodos, aos 16 e 29 dias após emergência (DAE), Eldorado do Sul - RS, 2005.

\begin{tabular}{|c|c|c|c|c|c|c|}
\hline \multirow[b]{2}{*}{ Cultivares } & \multicolumn{3}{|c|}{---------------------------16DAE--------------------------- } & \multicolumn{3}{|c|}{ 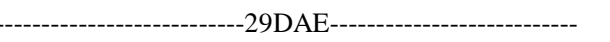 } \\
\hline & $\begin{array}{c}\text { Visual } \\
\text { (\%) }\end{array}$ & $\begin{array}{l}\text { Fotográfico } \\
\left(\mathrm{cm}^{2} \mathrm{~m}^{-2}\right)\end{array}$ & $\begin{array}{c}\text { Câmera de dossel } \\
\text { (\%) }\end{array}$ & $\begin{array}{c}\text { Visual } \\
\text { (\%) }\end{array}$ & $\begin{array}{l}\text { Fotográfico } \\
\left(\mathrm{cm}^{2} \mathrm{~m}^{-2}\right)\end{array}$ & $\begin{array}{c}\text { Câmera de dossel } \\
\text { (\%) }\end{array}$ \\
\hline 'ALBASUL' & $16,7 d^{1}$ & $338 \mathrm{e}$ & $18,0 \mathrm{c}$ & $38,5 \mathrm{ef}$ & $1077 \mathrm{~d}$ & $41,3^{\mathrm{ns}}$ \\
\hline 'CFT 1' & $24,4 \mathrm{ab}$ & $541 \mathrm{~cd}$ & $21,8 \mathrm{abc}$ & 51,4 abcd & $1432 \mathrm{abc}$ & 49,3 \\
\hline 'FAPA 5' & $21,2 \mathrm{bcd}$ & $454 d$ & 21,8 abc & 49,6bcd & $1533 \mathrm{ab}$ & 51,3 \\
\hline 'FAPA 6' & $23,1 \mathrm{abc}$ & $538 \mathrm{~cd}$ & $19,3 \mathrm{bc}$ & 49,3bcd & $1432 \mathrm{abc}$ & 44,5 \\
\hline 'UFRGS 19’ & $18,3 \mathrm{~cd}$ & $474 d$ & 19,8 bc & $30,6 f$ & $1202 \mathrm{~cd}$ & 32,3 \\
\hline 'UPF 16’ & $26,4 \mathrm{a}$ & $616 \mathrm{bc}$ & $27,4 \mathrm{a}$ & $61,8 \mathrm{a}$ & $1674 a$ & 64,9 \\
\hline ‘UPFA 20’ & $23,1 \mathrm{abc}$ & $510 \mathrm{~cd}$ & $22,8 \mathrm{abc}$ & $56,0 \mathrm{abc}$ & $1544 \mathrm{ab}$ & 54,5 \\
\hline 'UPFA 22’ & $25,6 \mathrm{ab}$ & $778 a$ & $27,0 \mathrm{a}$ & $59,8 \mathrm{ab}$ & $1702 \mathrm{a}$ & 56,9 \\
\hline ‘URS 21’ & $24,1 \mathrm{ab}$ & $663 b$ & 24,9ab & 48,5cde & $1474 \mathrm{ab}$ & 50,4 \\
\hline 'URS 22’ & 20,1bcd & $518 \mathrm{~cd}$ & $21,4 \mathrm{abc}$ & 42,5 de & $1300 \mathrm{bcd}$ & 44,5 \\
\hline Médias & 22,3 & 543 & 22,4 & 48,8 & 1437 & 49,0 \\
\hline CV (\%) & 13,9 & 13,6 & 16,7 & 13,5 & 13,5 & 25,2 \\
\hline
\end{tabular}

${ }^{1}$ Médias seguidas de letras distintas, comparadas nas colunas, diferem entre si pelo teste de Duncan $(\mathrm{P}=0,05)$. ${ }^{\mathrm{ns}} \mathrm{Não}$-significativo pelo teste $\mathrm{F}(\mathrm{P}=0,05)$.

Ciência Rural, v.39, n.5, ago, 2009. 
Neste estudo, foram usados três métodos para avaliar CS. No entanto, os métodos visual e fotográfico revelaram-se os mais precisos. Com a câmera de dossel, são obtidas leituras relativamente rápidas; contudo, o método visual ainda se mostra mais vantajoso, por ser rápido e prático de executar. Entretanto, segundo LOTZ et al. (1994), a experiência do observador ou as características das plantas podem influenciar os resultados do método visual. Nesse sentido, os observadores devem estar treinados para que o método possa ser confiável quando for utilizado para o manejo de plantas daninhas (VITTA \& FERNANDEZ QUINTANILLA, 1996). Por sua vez, a avaliação da CS pelo método fotográfico, que integra auxílios computacionais, representa alternativa promissora na quantificação da variável (RIZZARDI \& FLECK, 2004), embora seja mais trabalhoso e demorado para obtenção dos resultados.

Geralmente, as cultivares 'CFT 1' e 'UPF 16' apresentaram maiores EST em todas as avaliações (Tabela 2). Por outro lado, as cultivares 'ALBASUL', 'UFRGS 19' e 'URS 22' apresentaram os menores valores. Desse modo, 'CFT 1' e 'UPF 16' apresentam maior chance de captação de luz em estratos mais elevados do dossel da comunidade de plantas. No acúmulo de MSPA até os 16 DAE, a cultivar 'UPFA 20' superou as demais, exceto 'FAPA 5'. Por outro lado, 'UFRGS 19' posicionou-se no nível mais baixo, diferindo das demais. Já os maiores PC corresponderam às cultivares 'UPF 16' e 'UPFA 20', que superaram os demais genótipos; no entanto, esta última não diferiu de 'UPFA
22' e 'URS 21' (Tabela 2). Os menores índices de PC foram apresentados por 'ALBASUL' e 'UFRGS 19'. Nesse sentido, LEMERLE et al. (2001) relataram que cultivares de trigo com elevada habilidade competitiva com plantas daninhas apresentaram características como estatura elevada, folhas largas e decumbentes, crescimento vigoroso (estimado por meio da MSPA), abundante afilhamento e elevada cobertura do solo.

No presente estudo, as cultivares 'UPF 16' e 'UPFA 20' mostraram possuir características morfológicas vantajosas, dos pontos de vista de captação de luz e de potencial competitivo durante os primeiros 30 dias de crescimento da cultura. No entanto, isso não ocorreu com as cultivares 'ALBASUL' e 'UFRGS 19', as quais, em geral, não mostraram vantagens competitivas para características associadas ao crescimento das partes aéreas.

Os valores de MSPA na maturação e de PBA foram equivalentes entre as cultivares estudadas (Tabela 3). Maiores PG ocorreram em 'URS 21' e 'ALBASUL'; contudo, esta não diferiu de 'CFT 1' e 'FAPA6' (Tabela 3). Por outro lado, 'UPF 16' ' 'URS 22' apresentaram as menores PG, em valores absolutos. Por sua vez, os valores extremos para IC corresponderam às cultivares 'URS 21' (maior) e ‘UPF 16' (menor).

Os incrementos em EST, MSPA e CS entre 16 e 29 DAE aparecem na figura 1 . As cultivares 'FAPA 6' e 'UPF 16' apresentaram os maiores incrementos em EST. Por outro lado, 'URS 22' e 'UFRGS 19' apresentaram os menores incrementos para aquelas

Tabela 2 - População de plantas, estatura e matéria seca da parte aérea, avaliadas em três e em duas épocas, e potencial competitivo de plantas de cultivares de aveia, Eldorado do Sul - RS, 2005.

\begin{tabular}{|c|c|c|c|c|c|c|c|}
\hline \multirow{2}{*}{ Cultivares } & \multirow{2}{*}{$\begin{array}{l}\text { População } \\
\left(\text { plantas } \mathrm{m}^{-2} \text { ) }\right.\end{array}$} & \multicolumn{3}{|c|}{------------------Estatura (cm) ------------------ } & \multicolumn{2}{|c|}{ Matéria seca (mg planta ${ }^{-1}$ ) } & \multirow{2}{*}{$\begin{array}{l}\text { Potencial competitivo } \\
\text { (índice) }\end{array}$} \\
\hline & & $16 \mathrm{DAE}^{1}$ & $29 \mathrm{DAE}$ & $118 \mathrm{DAE}$ & $16 \mathrm{DAE}$ & $29 \mathrm{DAE}$ & \\
\hline 'ALBASUL' & $249 d^{2}$ & $18,3 \mathrm{c}$ & $27,2 \mathrm{~cd}$ & $100 \mathrm{c}$ & $73 b$ & $240^{\mathrm{ns}}$ & $2,9 \mathrm{f}$ \\
\hline ‘CFT 1’' & 340 abc & $21,5 \mathrm{a}$ & $31,0 \mathrm{ab}$ & $110 \mathrm{a}$ & $71 b$ & 240 & 3,5cdef \\
\hline 'FAPA 5' & $293 c d$ & 19,5 bc & 30,3ab & $107 a b$ & $77 \mathrm{ab}$ & 250 & 3,6cde \\
\hline 'FAPA 6' & $285 \mathrm{~cd}$ & 19,5 bc & $31,5 a b$ & $106 a b$ & $75 b$ & 230 & 3,3 def \\
\hline 'UFRGS 19’ & 316bcd & 19,3 bc & 25,7 de & $89 \mathrm{e}$ & $40 c$ & 190 & 3,1 ef \\
\hline ‘UPF 16’ & $403 a$ & $20,9 a b$ & $32,5 \mathrm{a}$ & $107 a b$ & $67 b$ & 260 & $4,9 a$ \\
\hline 'UPFA 20’ & $268 c d$ & $20,1 \mathrm{ab}$ & 29,9ab & $103 \mathrm{bc}$ & $92 \mathrm{a}$ & 280 & $4,5 a b$ \\
\hline 'UPFA 22’ & $376 a b$ & $19,9 \mathrm{abc}$ & $30,5 a b$ & $95 d$ & $67 b$ & 240 & 3,9bcd \\
\hline ‘URS 21’ & $329 \mathrm{abcd}$ & $20,3 a b$ & 29,3 bc & $104 \mathrm{bc}$ & $69 b$ & 220 & 4,1 bc \\
\hline ‘URS 22’ & $295 \mathrm{~cd}$ & $18,3 \mathrm{c}$ & $24,4 \mathrm{e}$ & 92 de & $67 b$ & 220 & 3,4def \\
\hline Médias & 315 & 19,8 & 29,2 & 101 & 70 & 240 & 3,7 \\
\hline CV (\%) & 15,4 & 5,0 & 5,7 & 2,9 & 14,7 & 16,8 & 5,9 \\
\hline
\end{tabular}

${ }^{1}$ Dias após a emergência. ${ }^{2}$ Médias seguidas de letras distintas, comparadas nas colunas, diferem entre si pelo teste de Duncan (P=0,05). ${ }^{\text {ns }}$ Não-significativo pelo teste $\mathrm{F}(\mathrm{P}=0,05) .{ }^{\text {ns }}$ Não-significativo em nível de $5 \%$ de probabilidade pelo teste $\mathrm{F}$.

Ciência Rural, v.39, n.5, ago, 2009. 
Tabela 3 - Matéria seca da parte aérea na maturação, produtividades biológica e de grãos e índice de colheita de plantas de cultivares de aveia, Eldorado do Sul - RS, 2005.

\begin{tabular}{|c|c|c|c|c|}
\hline Cultivares & $\begin{array}{l}\text { Matéria seca } \\
\left(\mathrm{kg} \mathrm{ha}^{-1}\right)\end{array}$ & $\begin{array}{l}\text { Produtividade Biológica } \\
\qquad\left(\mathrm{kg} \mathrm{ha}^{-1}\right)\end{array}$ & $\begin{array}{l}\text { Produtividade de grãos } \\
\qquad\left(\mathrm{kg} \mathrm{ha}^{-1}\right)\end{array}$ & $\begin{array}{l}\text { Índice de colheita } \\
\text { (\%) }\end{array}$ \\
\hline 'ALBASUL' & $6250^{\mathrm{ns}}$ & $10661^{\mathrm{ns}}$ & $5013 \mathrm{ab}^{1}$ & $47,2 \mathrm{ab}$ \\
\hline 'CFT 1' & 6750 & 11432 & $4812 \mathrm{abc}$ & 42,3abc \\
\hline 'FAPA 5' & 7200 & 11543 & 4166 cde & 36,2 bc \\
\hline 'FAPA 6' & 6650 & 10985 & 4507 bcd & $42,2 \mathrm{abc}$ \\
\hline 'UFRGS 19' & 5450 & 8827 & 3877 def & $44,2 \mathrm{abc}$ \\
\hline ‘UPF 16’ & 6250 & 10138 & $3240 \mathrm{f}$ & $33,2 \mathrm{c}$ \\
\hline 'UPFA 20’ & 6600 & 11172 & 4000 de & 36,8 bc \\
\hline 'UPFA 22’ & 7125 & 11987 & 4222 cde & 35,7 bc \\
\hline ‘URS 21’ & 6450 & 10821 & $5206 a$ & $48,6 a$ \\
\hline ‘URS 22’ & 5825 & 9329 & $3746 \mathrm{ef}$ & $42,1 \mathrm{abc}$ \\
\hline Médias & 6455 & 10689 & 4279 & 40,8 \\
\hline CV (\%) & 14,9 & 15,6 & 10,4 & 17,0 \\
\hline
\end{tabular}

${ }^{\text {ns }}$ Não-significativo pelo teste $\mathrm{F}(\mathrm{P}=0,05) .{ }^{1}$ Médias seguidas de letras distintas, comparadas nas colunas, diferem entre si pelo teste de Duncan $(\mathrm{P}=0,05)$.

variáveis. Para CS, destacaram-se 'UPF 16' e 'UPFA 22', com os maiores incrementos. Já 'URS 21', 'URS 22' e 'ALBASUL' demonstraram baixos incrementos durante o período avaliado. Em parte, os resultados obtidos para as variáveis estudadas podem ter sido influenciados por diferenças nas populações de plantas das cultivares (Tabela 2). Elevadas CS e EST, aliadas aos rápidos incrementos nessas variáveis, proporcionariam às cultivares formação de dosséis densos que poderiam reduzir a penetração de luz dentro da comunidade vegetal.

Algumas cultivares demonstraram, a princípio, baixo potencial competitivo na fase inicial do ciclo. Por exemplo, a cultivar 'ALBASUL' apresentou baixas EST e CS aos 16 e 29 DAE e, também, lento incremento em CS e baixo PC. A cultivar 'UFRGS 19' também respondeu desfavoravelmente, mostrando baixa EST aos 29DAE e, no final do ciclo, baixa CS aos 16 e 29DAE. Além disso, essa cultivar demonstrou lentos incrementos, tanto em EST, quanto em CS, além de baixo PC. Para 'URS 22', foram observadas baixas EST aos 16 e 29 DAE e também no final do ciclo. Além disso, foram observadas baixas CS aos 16 e 29DAE e lentos incrementos em EST e em CS, além de reduzido PC. Embora a cultivar 'UPF 16’ apresentasse nítido perfil competidor, ou o contrário, 'ALBASUL' deixasse a desejar nesse aspecto, foram observados resultados divergentes entre ambas quando se comparam PG e IC. Assim, 'UPF 16', de alto PC inicial, mostrou baixos PG e IC. Já 'ALBASUL', um genótipo que pode ser classificados como de baixo PC inicial, apresentou elevada PG e alto IC.
O estudo procurou investigar a variabilidade de características morfológicas em plantas de cultivares de aveia no início do ciclo, na ausência de plantas concorrentes. Nesse contexto, três aspectos devem ser considerados. Primeiro: características vantajosas à capacidade de competição, identificadas nos estádios iniciais, podem não se sustentarem como tal durante todo o ciclo. Segundo: em presença de plantas infestantes, podem ocorrer adaptações e compensações entre características apontadas como típicas de cultivar competidora, de modo a modificar o contexto de relacionamento entre as espécies durante as diferentes fases do ciclo. Terceiro: uma cultivar definida na fase inicial como de baixo potencial de competição pode compensar tal deficiência por meio de elevado potencial produtivo de grãos ('ALBASUL'), o qual poderá superar aquela limitação, mas o inverso também pode ocorrer ('UPF 16'). Consequentemente, é importante a realização de trabalhos que possam elucidar as dúvidas originadas no estudo inicial.

\section{CONCLUSÕES}

Cultivares de aveia mostram variações em características morfológicas durante os estádios iniciais de desenvolvimento que podem se associar à habilidade competitiva com plantas daninhas. Na fase inicial do ciclo e na ausência de plantas concorrentes, as cultivares 'UPF 16' e 'UPFA 22' apresentam características morfológicas que as identificam como competidoras potenciais. Já a cultivar ‘ALBASUL' não mostra ser portadora de atributos de cultivar competidora. Os métodos visual, fotográfico e câmera 


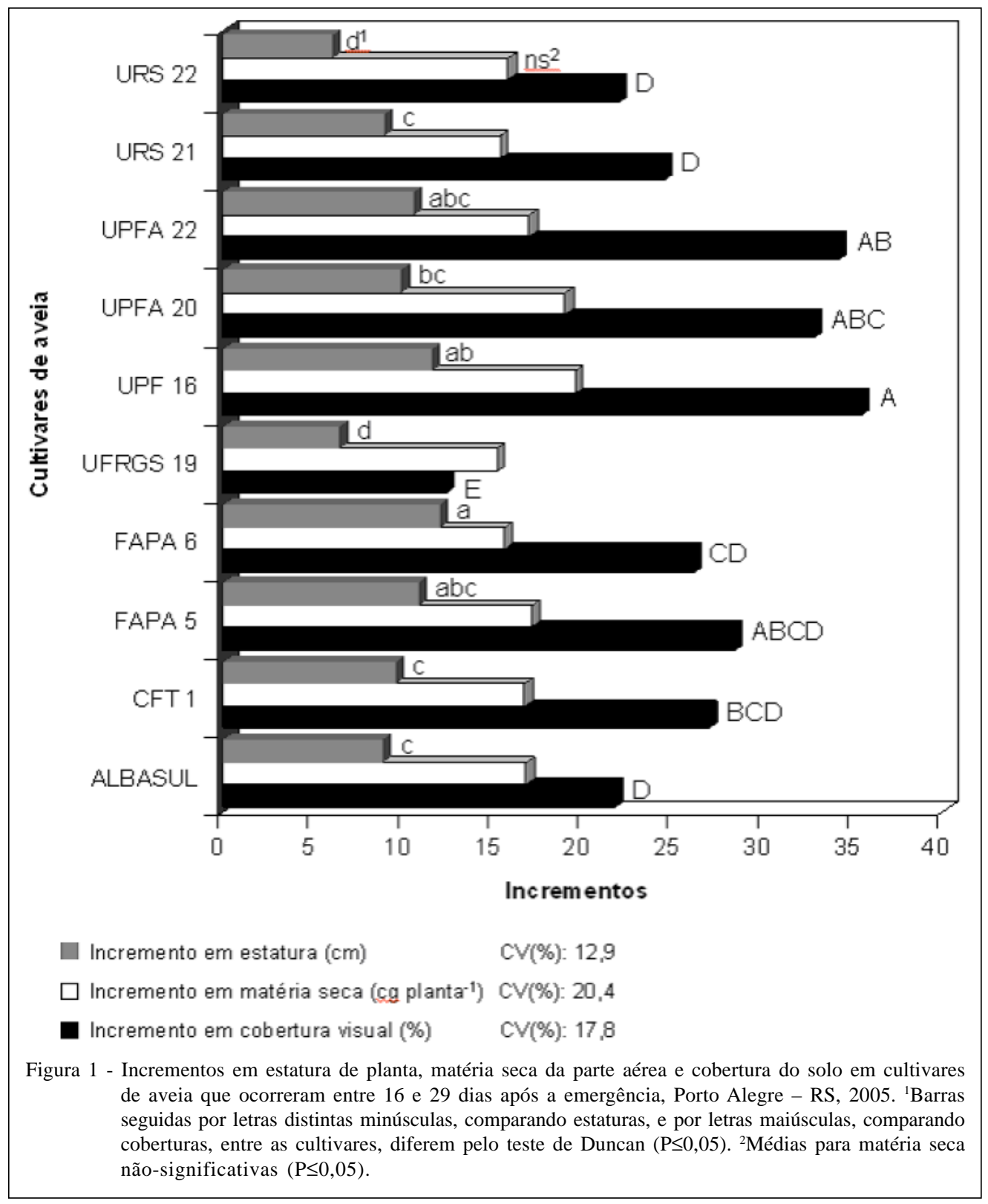

de dossel são viáveis para avaliar cobertura do solo projetada pelo dossel de aveia. No entanto, os métodos visual e fotográfico apresentam menores variabilidades, mas o primeiro é mais rápido e prático de executar.

\section{REFERÊNCIAS}

BALBINOT JR. A.A. et al. Velocidade de emergência e crescimento inicial de cultivares de arroz irrigado influenciando a competitividade com as plantas daninhas. Planta Daninha, Viçosa, v.19, n.3, p.305-316, 2001.

BLACKSHAW, R.E. Differential competitive ability of winter wheat cultivars against downy brome. Agronomy Journal, Madison, v.86, n.4, p.649-654, 1994.
FLECK, N.G. Competição de azevém (Lolium multiflorum L.) com duas cultivares de trigo. Planta Daninha, Piracicaba, v.3, n.2, p.61-67, 1980.

GOLDBERG, D.E.; LANDA, K. Competitive effect and response: hierarchies and correlated traits in the early stages of competition. Journal of Ecology, Oxford, v.79, n.4, p.10131030, 1991.

HAEFELE, S.M. et al. Field screening of diverse rice genotypes for weed competitiveness in irrigated lowland ecosystems. Field Crops Research, Amsterdam, v.88, n.1, p.39-56, 2004. Disponível em: <http://www.sciencedirect.com/ science?_ob=ArticleURL\&_udi=B6T6M-4BJ778S2 \&_user $=687304 \&$ _coverDate $=06 \%$ 2F10\%2F2004\&_rdo $\mathrm{c}=6 \& \&_{-} \mathrm{fmt}=\mathrm{high} \& \_$orig $=$browse \&_srch $=$doc -

Ciência Rural, v.39, n.5, ago, 2009. 
info(\%23toc\%235034\%232004\%23999119998\%23497053\% 23 F L A \%23 display\%23 Volume) \& cdi $=5034$ \& sort $=$ d\&_docanchor $=\&$ \&_ct $=9 \&$ \&_acct $=$ C $0000377 \overline{9}$ $8 \&$ \&_versio n $=1 \&$ \& u r l Versio $n=0 \&$ _ u s e ri d

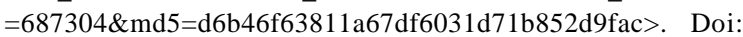
10.1016/j.fcr.2003.11.010.

LEMERLE, D. et al. Genetic improvement and agronomy for enhanced wheat competitiveness with weeds. Australian Journal of Agricultural Research, Oxford, v.52, n.1, p.527548, 2001. Disponível em: <http://www.publish.csiro.au/nid/ 40/paper/AR00056.htm>. Doi: 10.1071/AR00056.

LEMERLE, D. et al. Incremental crop tolerance to weeds: A measure for selecting competitive ability in Australian wheats. Euphytica, Dordrecht, v.149, n.1-2, p.85-95, 2006. Disponível em: <http://www.springerlink.com.w10048.dotlib.com.br/

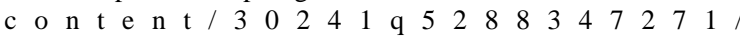
?p=ed7dfa0c4b8f45aa8338ec943dbd800e\&pi=9>. Doi: $10.1007 / \mathrm{s} 10681-005-9056-5$.

LOTZ, L.A.P. et al. Techniques to estimate relative leaf area and cover of weeds in crops for yield loss prediction. Weed Research, Oxford, v.34, n.3, p.167-175, 1994. Disponível em: <http://www3.interscience.wiley.com/journal/119268463/ abstract>. Doi: 10.1111/j.1365-3180.1994.tb01984.x.
NI, H. et al. Oryza sativa plant traits conferring competitive ability against weeds. Weed Science, Lawrence, v.48, n.2, p.200-204, 2000. Disponível em: <http://www.bioone.org/ archive/0043-1745/48/2/pdf/i0043-1745-48-2-200.pdf >. Doi: 10.1614/0043-1745(2000)048[0200:OSPTCC]2.0.CO;2.

RADOSEVICH, S. et al. Weed ecology: implications for management. In:__. Physiological aspects of competition. 2.ed. New York: Willey, 1997. Cap. 6, p.217-299.

RIZZARDI, M.A.; FLECK, N.G. Métodos de quantificação da cobertura foliar da infestação de plantas daninhas e da cultura da soja. Ciência Rural, Santa Maria, v.34, n.1, p.13-18, 2004. Disponível em: <http://www.scielo.br/ scielo.php ? s c ri pt = sci_art text \& pid = S 0103 $84782004000100003 \& \operatorname{lng}=\mathrm{en} \& \mathrm{nrm}=\mathrm{i}$ so\&tlng $=\mathrm{pt}>$. Doi: $10.1590 / \mathrm{S} 0103-84782004000100003$.

VITTA, J.I.; FERNANDEZ QUINTANILLA, C. Canopy measurements as predictors of weed-crop competition. Weed Science, Champaign, v.44, n.3, p.511-516, 1996.

WICKS, G.A. et al. Winter wheat cultivar characteristics affect annual weed suppression. Weed Technology, Lawrence, v.18, n.4, p.988-998, 2004. Disponível em: <http://www.bioone.org/ perlserv/?request $=$ get-abstract $\&$ doi $=10.1614 \% 2$ FWT-03158R1>. Doi: 10.1614/WT-03-158R1. 